\title{
Face Recognition by Neural Network Using Bit- planes Extracted from An Image
}

Article in Journal of Information and Computational Science · November 2013

DOI: $10.12733 / \mathrm{jics} 20102079$

CITATIONS

3

2 authors, including:

\section{James Tan}

SARAWAK ENERGY BERHAD

4 PUBLICATIONS 14 CITATIONS

SEE PROFILE
READS

9 


\title{
Face Recognition by Neural Network Using Bit-planes Extracted from An Image
}

\author{
K. C. Ting, J. Y. B. Tan* ${ }^{*}$ T. Z. Lee, D. B. L. Bong \\ Faculty of Engineering, Universiti Malaysia Sarawak, 94300 Kota Samarahan, Sarawak, Malaysia
}

\begin{abstract}
An 8-bit digital image consists of 256 levels of gray-value and 8 layers of multilevel information of bits known as bit-plane information. A novel method utilizing higher order bit-plane information that contains majority of visually significant data and dummy blank images as inputs to a multilayer feedforward Neural Network (NN) is proposed in this paper to perform face recognition. Experiments performed on the proposed face recognition model using two face databases, namely CMU AMP face expression database and Yale face database, show improvement in recognition rate compared to using only gray-level images as inputs to the NN.
\end{abstract}

Keywords: Bit-plane; Face Recognition; Image Processing; Neural Network

\section{Introduction}

Face recognition technology is researched extensively due to its potential for use in various applications such as content-based indexing, information security and law enforcement [1]. Much of the work in face recognition in the past focused on detecting individual features such as the eyes, nose, mouth and head outline, and define a face model by the position, size, and relationships among these features. However, such approaches have often been quite fragile [2]. Currently, various techniques are widely used for face feature extraction. Examples of these methods include Gabor analysis [3], Elastic Brunch Matching [4], Line Edge Map [5] and multivariate analysis method-

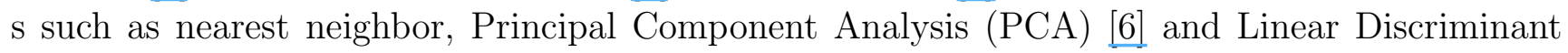
Analysis (LDA). However these methods utilize complex mathematical calculations and provide only single feature extraction.

A digital image is constructed using multilevel information of bits, called as bit-plane information. Bit-plane information, which contain useful raw data of image, is useful in image coding especially in image compression $[7,8]$. A novel feature extraction method of manipulating bitplane information to act as inputs to neural network is proposed in this paper. Unlike conventional methods, which involve highly complex mathematical calculations and only provide single feature

${ }^{*}$ Corresponding author.

Email address: yiawbeng@yahoo.com (J. Y. B. Tan). 